\title{
Dynamic analysis of pulmonary computed tomography (CT) characteristics in cured coronavirus disease 2019 (COVID-19) patients
}

\author{
Yunhui Yang ${ }^{1 \#}$, Zhenghua Zhang ${ }^{2 \#}$, Yuanming Jiang ${ }^{2 *}$, Zhipeng $\mathrm{Li}^{3}$, Hongyuan Yang ${ }^{4}, \mathrm{Zhi} \mathrm{Li}^{5}, \mathrm{Xiang}^{{ }^{6}}{ }^{6}$, \\ Juntao Yang ${ }^{7}$, Jing Zhang ${ }^{8}$, Yan Peng ${ }^{9}$, Lin Luo ${ }^{10}$, Wen Chang ${ }^{10}$, Wei Zhao ${ }^{11}$, Yi Wu ${ }^{12}$, Cunwen Ma ${ }^{13}$, \\ Shiping Wang ${ }^{14}$, Yongjian Wen ${ }^{15}$, Fushun Pu ${ }^{16}$, Xuefei Jiang ${ }^{17}$, Lishuang Shen ${ }^{18}$, Zhengyi Zhan ${ }^{19}$, Yong Jin ${ }^{20}$, \\ Qiming $\mathrm{Li}^{21}$, Wenfang $\mathrm{Yi}^{2}$, Ying Xie ${ }^{22}$, Yanlong Tang ${ }^{23}$, Hao Duan ${ }^{24}$, Kai Yang ${ }^{25}$, Yu Su ${ }^{26}$, Chuncai $\mathrm{Wu}^{27}$, \\ Zhongzheng Luo ${ }^{28}$, Peng Wang ${ }^{29}$, Minchang Hong ${ }^{9}$, Shiqiang Yang ${ }^{30}$, Wenyong Bai ${ }^{31}$, Guilin $\mathbf{Y u}^{32}$, \\ Shaohua Liu ${ }^{33}$, Zhongmin $\mathrm{Nie}^{34}$, Guangbi Song ${ }^{35}$, Dongquan Guo ${ }^{36}$, Xiaobo He ${ }^{37}$, Zhiyu Chen ${ }^{11}$, \\ Kaiyi Xue ${ }^{38}$, Jiyao $\mathrm{Ma}^{2}$, Yuchun $\mathrm{Wu}^{39}$, Xiaotao Yang ${ }^{40}$, Bo $\mathrm{He}^{2}$, Kunhua Wang ${ }^{41}$, Jiangyuan $\mathrm{Pi}^{42}$
}

${ }^{1}$ Department of Medical Imaging, People's Hospital of Xishuangbanna Dai Autonomous Prefecture, Jinghong, China; ${ }^{2}$ Medical Imaging Department, First Affiliated Hospital of Kunming Medical University, Kunming, China; ${ }^{3}$ Medical Imaging Department, Yunnan Provincial Infectious Disease Hospital, also named Yunnan AIDS Care Center, Kunming, China; ${ }^{4}$ Radiology Department, Lijiang People's Hospital, Kunming, China; ${ }^{5}$ Department of Radiology, The First People's Hospital of Yunnan Province, Kunming, China; ${ }^{6}$ Department of Radiology, The Third Peoples' Hospital of Kunming, Kunming, China; 'Department of Radiology, Dali Bai Autonomous Prefecture People's Hospital, Dali, China; ${ }^{8}$ CT Room, People's Hospital of Yuxi City, Yuxi, China; ${ }^{9}$ Department of Radiology, the First People's Hospital of Zhaotong, Zhaotong, China; ${ }^{10}$ Department of Radiology, The First People's Hospital of Quqin City, Quqin, China; ${ }^{11}$ Department of Radiology, Baoshan People's Hospital, Baoshan, China; ${ }^{12}$ Medical Imaging Department, Chuxiong People's Hospital, Chuxiong, China; ${ }^{13}$ Department of Radiology, the People's Hospital of Wenshan Zhuang and Miao Minority Autonomous Prefecture, Wenshan, China; ${ }^{14}$ Department of Radiology, Kungang Hospital of Yunnan, Kungang, China; ${ }^{15}$ Radiology Department, the Third People's Hospital of Yunnan Province, Kunming, China; ${ }^{16}$ Medical Imaging Department, the First People's Hospital of Honghe State, Mengzi, China; ${ }^{17}$ Department of Medical Imaging, Weixin County People's Hospital, Zhaotong, China; ${ }^{18}$ Department of Medical Imaging, Jianshui County People’s Hospital, Mengzi, China; ${ }^{19}$ Department of Medical Imaging, Daguan County People's Hospital, Zhaotong, China; ${ }^{20}$ Medical Imaging Department, Pu'er Peoples' Hospital of Yun Nan, Pu'er, China; ${ }^{21}$ Department of Radiology, the First People's Hospital of Lancang County, Pu'er, China; ${ }^{22}$ Calmette Hospital and The First Hospital of Kunming, Kunming, China; ${ }^{23}$ Radiology Department, the First Affiliated Hospital of Dali University, Dali, China; ${ }^{24}$ Medical Imaging Department, Binchuan People's Hospital, Binchuan, China; ${ }^{25}$ Medical Imaging Department, Vice President of the Dali City Second People's Hospital, Dali, China; ${ }^{26}$ Department of Radiology, NO.1 People's Hospital of Dali City, Dali, China; ${ }^{27}$ Medical Imaging Department, Yongshan People's Hospital; China; ${ }^{28}$ Department of Medical Imaging, Qiaojia County People's Hospital, Zhaotong, China; ${ }^{20}$ Radiology Department, the Second People's Hospital of Yunnan Province, Kunming, China; ${ }^{30}$ Department of Medical Imaging, Ruili County People's Hospital, Ruili, China; ${ }^{31}$ Department of Radiology, the First People's Hospital of Xuanwei City, Xuanwei, China; ${ }^{32}$ Department of Medical Imaging, Lvliang County People's Hospital, Lvliang, China; ${ }^{33}$ Department of Radiology, Qilin District People's Hospital of Qujing City, Qujing, China; ${ }^{34}$ Medical Imaging Department, Huize People's Hospital, Huize, China; ${ }^{35}$ Medical Imaging Center, Luoping County People's Hospital, Qujing, China; ${ }^{36}$ Department of Medical Imaging, Chengjiang County People's Hospital, Chengjiang, China; ${ }^{37}$ Department of Radiology, Kunming Jingkai People's Hospital, Kunming, China; ${ }^{38}$ The North District Branch of Kunming Maternal and Child Health Hospital, Kunming, China; ${ }^{39}$ Medical Imaging Department, the First People's Hospital of Xundian County, Kunming, China; ${ }^{40}$ Department of Radiology, Yunnan Xinxinhua Hospital, Kunming, China; ${ }^{41}$ NHC Key Laboratory of Drug Addiction Medicine, First Affiliated Hospital of Kunming Medical University, Kunming Medical University, Kunming, China; ${ }^{42}$ Kunming Medical University, Kunming, China

Contributions: (I) Conception and design: Z Zhang, B He; (II) Administrative support: Y Yang, B He, K Wang, J Pi; (III) Provision of study materials or patients: Z Li, H Yang, X Li, J Yang, J Zhang, Y Peng, L Luo, W Chang, W Zhao, Y Wu, C Ma, S Wang, Y Wen, F Pu, X Jiang, L Shen, Z Zhan, Y Jin, Q Li, W Yi, Y Xie, Y Tang, H Duan, K Yang, Y Su, C Wu, Z Luo, P Wang, M Hong, S Yang, W Bai, G Yu, S Liu, Z Nie, G Song, D Guo, X He, Z Chen, K Xue, J Ma, Y Wu, X Yang ; (IV) Collection and assembly of data: Z Zhang, Y Yang, Y Jiang; (V) Data analysis and interpretation: Z Zhang, Y Yang, Y Jiang; (VI) Manuscript writing: All authors; (VII) Final approval of manuscript: All authors.

"These authors contributed equally to this work.

Correspondence to: Bo He, MD. NHC Key Laboratory of Drug Addiction Medicine, Medical Imaging Department, First Affiliated Hospital of 
Kunming Medical University, Kunming Medical University, Kunming 650032, China. Email: hebo_ydyy@qq.com; Kunhua Wang, MD. NHC Key Laboratory of Drug Addiction Medicine, First Affiliated Hospital of Kunming Medical University, Kunming Medical University, Kunming 650032, China. Email: wangkunhua_ydyy@163.com; Jiangyuan Pi, MM. Kunming Medical University, No. 1168 Chunrongxi Road Chenggong District, Kunming 650500, China. Email: 493335833@qq.com.

Background To retrospectively analyze the pulmonary computed tomography (CT) characteristics and dynamic changes in the lungs of cured coronavirus disease 2019 (COVID-19) patients at discharge and reexamination.

Methods: A total of 155 cured COVID-19 patients admitted to designated hospitals in Yunnan Province, China, from February 1, 2020, to March 20, 2020, were included. All patients underwent pulmonary CT at discharge and at 2 weeks after discharge (during reexamination at hospital). A retrospective analysis was performed using these two pulmonary CT scans of the cured patients to observe changes in the number, distribution, morphology, and density of lesions.

Results: At discharge, the lung CT images of 15 cured patients showed no obvious lesions, while those of the remaining 140 patients showed different degrees of residual lesions. Patients with moderate disease mostly had multiple pulmonary lesions, mainly in the lower lobes of both lungs. At reexamination, the lung lesions in the patients with moderate disease had significantly improved $(\mathrm{P}<0.05)$, and the lung lesions in the patients with severe disease had partially improved, especially in patients with multi-lobe involvement $\left(\chi^{2}=3.956, \mathrm{P}<0.05\right)$. At reexamination, the lung lesions of patients with severe disease did not show significant changes $(\mathrm{P}>0.05)$.

Conclusions: The pulmonary CT manifestations of cured COVID-19 patients had certain characteristics and variation patterns, providing a reference for the clinical evaluation of treatment efficacy and prognosis of patients.

Keywords: Severe acute respiratory syndrome coronavirus 2 (SARS-CoV-2); pneumonia; computed tomography (CT); X-ray computed tomography (X-ray CT)

Submitted Nov 19, 2020. Accepted for publication Jan 26, 2021.

doi: 10.21037/apm-20-2532

View this article at: http://dx.doi.org/10.21037/apm-20-2532

\section{Introduction}

Since December 2019, pneumonia caused by the severe acute respiratory syndrome coronavirus 2 (SARS-CoV-2) has spread throughout China and around the world, resulting in serious impacts on people's health and life, society, and the economy $(1,2)$. On February 7, 2020, the National Health Council of China changed the name of novel coronavirus pneumonia to coronavirus disease 2019 (COVID-19), whose primary manifestations are fever, dry cough, and fatigue. A COVID-19 diagnosis requires a positive nucleic acid test result or viral gene sequencing (3). Due to differences in the specificity and sensitivity of the tests, false negative results are prone to occur. Thus, imaging has become a convenient and effective means for the diagnosis and assessment of COVID-19, and there have been some studies on the pulmonary imaging characteristics of COVID-19 patients (4-6).
With the implementation of prevention and control measures, as well as relevant effective treatments, COVID-19 patients have been cured and discharged extensively nationwide. However, the healing process and patterns of COVID-19 patients are still unclear. Some scholars have summarized the pulmonary computed tomography (CT) characteristics of COVID-19 patients during hospitalization $(7,8)$, but the imaging characteristics and outcomes in the lungs of cured COVID-19 patients have not been reported. Therefore, this study retrospectively analyzed the pulmonary CT characteristics and dynamic changes in the lungs of cured COVID-19 patients at discharge and reexamination to provide a reference for the clinical assessment of treatment efficacy and patient prognosis. Observing the short-term residual and change of lung lesions after cure is beneficial to the clinical understanding of the absorption rule of lung lesions 
Table 1 Basic information of coronavirus disease 2019 (COVID-19) patients

\begin{tabular}{|c|c|}
\hline Clinical features & Patient $(n=155)$ \\
\hline \multicolumn{2}{|l|}{ Age } \\
\hline Median & 41 \\
\hline Range & $8-73$ \\
\hline$<41$ & $78(50.3 \%)$ \\
\hline$\geq 41$ & $77(49.7 \%)$ \\
\hline \multicolumn{2}{|l|}{ Sex } \\
\hline Male & $76(49.0 \%)$ \\
\hline Female & $79(51.0 \%)$ \\
\hline \multicolumn{2}{|l|}{ Exposure history } \\
\hline Hubei residence history & $95(61.3 \%)$ \\
\hline Close contact & $60(38.7 \%)$ \\
\hline \multicolumn{2}{|l|}{ Clinical symptoms } \\
\hline Fever & $132(85.2 \%)$ \\
\hline Cough & $129(83.2 \%)$ \\
\hline Shortness of breath & $16(10.3 \%)$ \\
\hline Sore throat & $41(26.5 \%)$ \\
\hline Running nose & $62(40.0 \%)$ \\
\hline Fatigue & $123(79.4 \%)$ \\
\hline Headache & $50(32.3 \%)$ \\
\hline Muscular soreness & $14(9.0 \%)$ \\
\hline Chest pain & $11(7.1 \%)$ \\
\hline Nausea, vomiting, and diarrhea & $17(11.0 \%)$ \\
\hline No obvious clinical manifestations & $14(9.0 \%)$ \\
\hline \multicolumn{2}{|l|}{ Comorbidities } \\
\hline Acute respiratory distress syndrome (ARDS) & $1(0.6 \%)$ \\
\hline Acute kidney injury & $1(0.6 \%)$ \\
\hline Acute liver injury & $1(0.6 \%)$ \\
\hline \multicolumn{2}{|l|}{ Underlying disease } \\
\hline Diabetes & $9(5.8 \%)$ \\
\hline Hypertension & $8(5.2 \%)$ \\
\hline $\begin{array}{l}\text { Chronic obstructive pulmonary disease } \\
\text { (COPD) }\end{array}$ & $3(1.9 \%)$ \\
\hline Coronary heart disease & $1(0.6 \%)$ \\
\hline Hepatitis B & $2(1.3 \%)$ \\
\hline
\end{tabular}

Table 1 (continued)
Table 1 (continued)

\begin{tabular}{lc}
\hline Clinical features & Patient $(\mathrm{n}=155)$ \\
\hline Hypothyroidism & $1(0.6 \%)$ \\
Hyperthyroidism & $1(0.6 \%)$ \\
Clinical classification & \\
Moderate & $135(87.1 \%)$ \\
Severe & $20(12.9 \%)$ \\
\hline
\end{tabular}

after treatment.

We present the following article in accordance with the MDAR checklist (available at http://dx.doi.org/10.21037/ apm-20-2532).

\section{Methods}

\section{General information}

A total of 155 cured COVID-19 patients admitted to designated hospitals in Yunnan Province, China, from February 1, 2020, to March 20, 2020, were included in the present study. The patients' relevant basic information is shown in Table 1. The diagnosis and discharge standards of all patients were in line with the Diagnosis and Treatment of COVID-19 ( $7^{\text {th }}$ edition) guidelines. According to the guidelines, all patients underwent pulmonary CT at discharge and at 2 weeks after discharge. All procedures performed in this study involving human participants were in accordance with the Declaration of Helsinki (as revised in 2013). This study was approved by the Ethics Committee of First Affiliated Hospital of Kunming Medical University, and confirm that informed consent was obtained from all patients.

\section{Instruments and methods}

Included patients underwent pulmonary CT in a specific room at discharge and at 2 weeks after discharge; the room was strictly disinfected before and after examination, and all technicians used appropriate personal protective equipment. A 64-slice spiral CT (Philips, Netherlands; GE, USA; and Siemens, Germany) was used, and the scanning parameters were as follows: tube voltage, $100-120 \mathrm{kV}$; tube current, 100-300 mA; scan slice thickness, $5 \mathrm{~mm}$; slice thicknesses for image reconstruction, $1 \mathrm{~mm}$; spacing, $0.7 \mathrm{~mm}$ (window width: 1,500 $\mathrm{HU}$, window position: $-400 \mathrm{HU}$ ); and scan 
Table 2 Distribution of lung lesions in patients with moderate and severe disease at discharge and reexamination

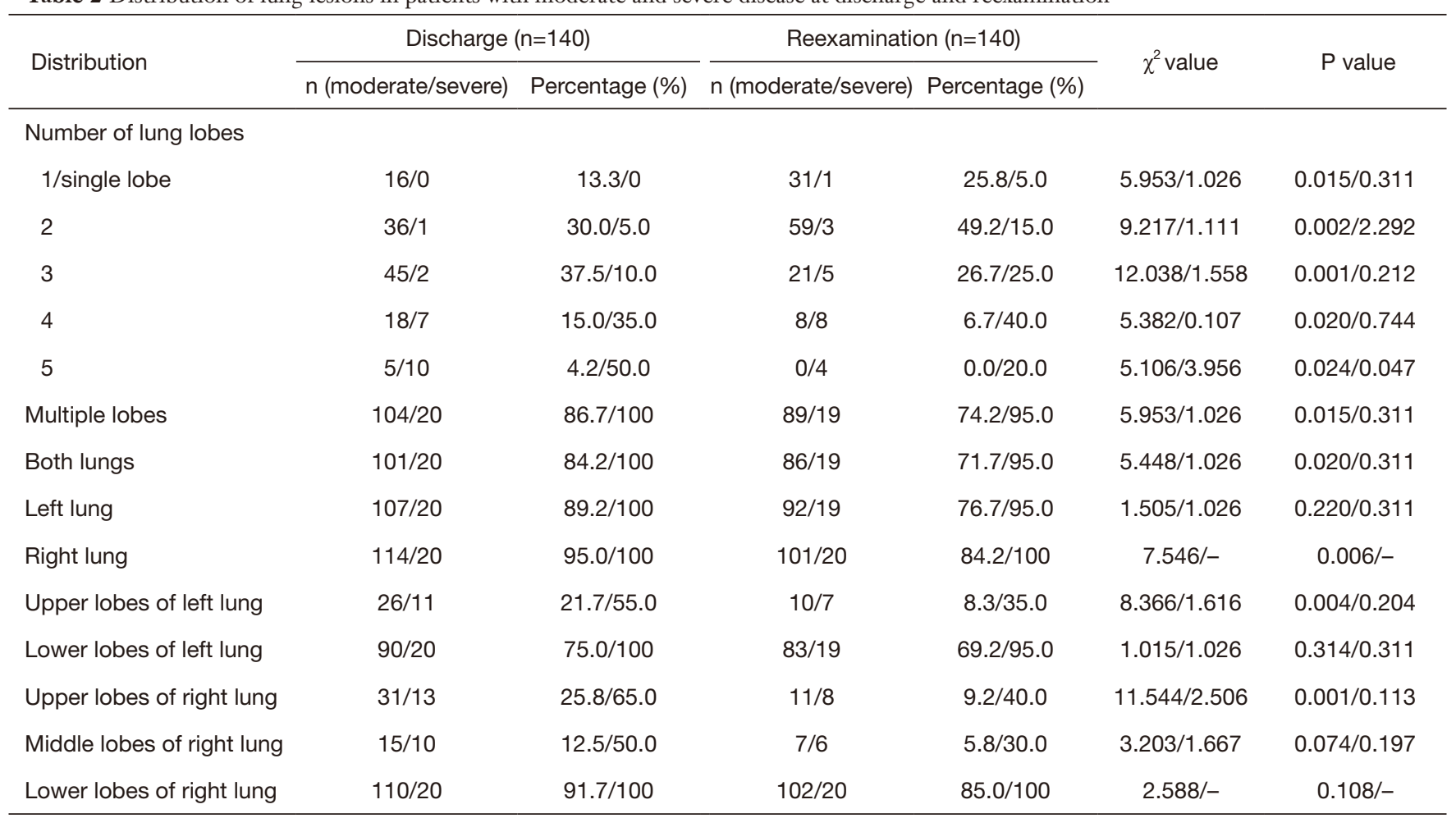

range, from the base to the tip of the lung.

Two associate chief radiologists with experience in chest imaging were selected to examine the images independently. When there was disagreement between these two radiologists, a third senior radiologist examined the images and made the final decision. Image analysis included lesion distribution, morphology, ground-glass opacities (GGOs), consolidation shadows, interlobular septal thickening (IST), parenchymal bands, subpleural lines (dense linear shadows parallel to the pleura), vascular enlargement in lesions, and air bronchograms.

\section{Statistical methods}

SPSS Statistics 20.0 (IBM, USA) was used for statistical analysis, and Pearson's chi-square test was also used. Count data were expressed as the number of cases and the percentage [n (\%)], and $\mathrm{P}<0.05$ was considered statistically significant.

\section{Results}

\section{Pulmonary CT characteristics at discharge}

Fifteen cured patients did not have obvious lesions on pulmonary CT at discharge and did not return to the hospital for reexamination. Based on pulmonary CT images, the remaining 140 patients still had various degrees of residual lesions at discharge, which were mainly distributed in the periphery and subpleural area of both lungs, especially in the lower lobes of the lungs.

Multiple pulmonary lesions were the main manifestations in patients with moderate disease (104 cases, 86.7\%), primarily in the lower lobes of both lungs (Table 2). For these patients, the main pulmonary CT manifestations were as follows: GGOs in 38 patients (31.7\%), mostly in the shape of a patch or strip; consolidation shadows in 82 patients $(68.3 \%)$, mainly patchy or irregular shapes; air bronchograms in 11 patients (9.2\%); IST in 92 patients (76.7\%) and parenchymal bands in 111 patients (92.5\%), observed in primary lesions; dense linear shadows parallel to the pleura (subpleural lines) in 86 patients (71.7\%), observed in lesions in the periphery of both lungs; and vascular enlargement in lesions in 26 patients (21.7\%) (Table 3). No obvious pleural effusion or lymph node enlargement was observed.

Patients with severe disease mostly had multiple pulmonary lesions that involved multiple lobes (Table 2). 
Table 3 Computed tomography (CT) characteristics of patients with moderate disease at discharge and reexamination

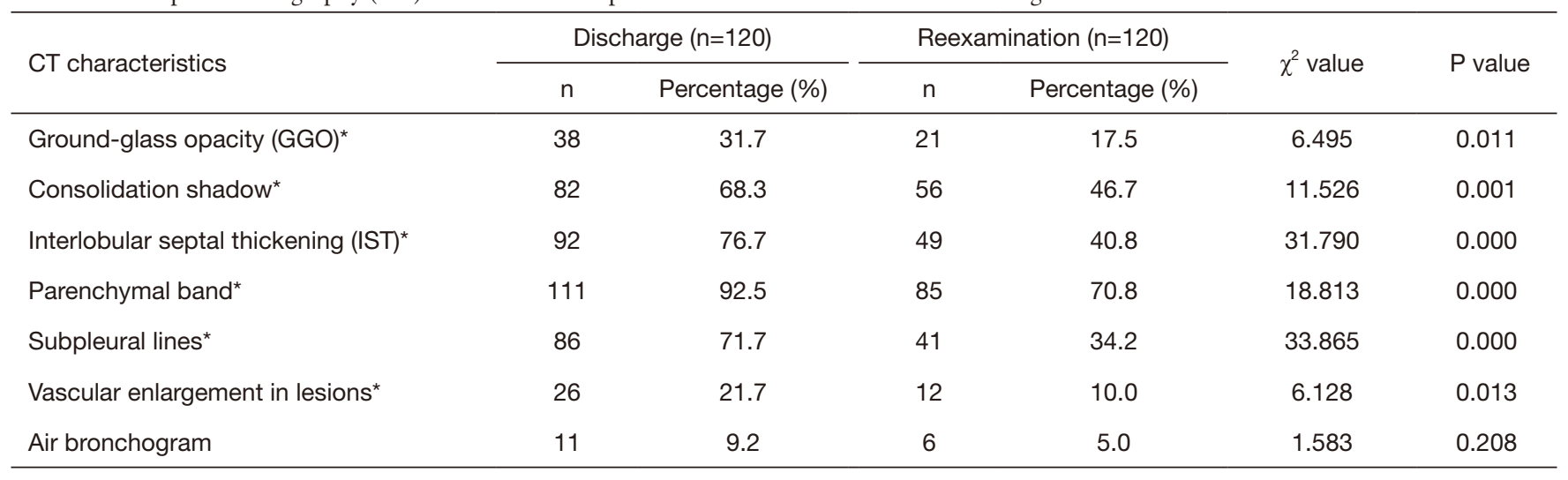

*, $\mathrm{P}<0.05$.

Table 4 Computed tomography (CT) characteristics of patients with severe disease at discharge and reexamination

\begin{tabular}{|c|c|c|c|c|c|c|}
\hline CT characteristics & \multicolumn{2}{|c|}{ Discharge $(n=20)$} & \multicolumn{2}{|c|}{ Reexamination $(n=20)$} & $\chi^{2}$ value & $P$ value \\
\hline Ground-glass opacity (GGO) & 19 & 95 & 18 & 90 & 0.360 & 0.548 \\
\hline Consolidation shadow & 10 & 50 & 9 & 45 & 0.100 & 0.752 \\
\hline Interlobular septal thickening (IST) & 16 & 80 & 13 & 65 & 1.129 & 0.288 \\
\hline Subpleural lines & 6 & 30 & 8 & 40 & 0.440 & 0.507 \\
\hline Vascular enlargement in lesions & 10 & 50 & 6 & 30 & 1.667 & 0.197 \\
\hline Air bronchogram & 4 & 20 & 3 & 15 & 0.173 & 0.677 \\
\hline
\end{tabular}

The main pulmonary CT manifestations in these patients were as follows: GGOs in 19 patients $(95.0 \%)$, consolidation shadows in 10 patients $(50.0 \%)$, IST in 16 patients $(80.0 \%)$, parenchymal bands in 15 patients $(75.0 \%)$, subpleural lines in six patients $(30.0 \%)$, vascular enlargement in lesions in 10 patients $(50.0 \%)$, and air bronchograms in 4 patients (20.0\%) (Table 4). No obvious pleural effusion or lymph node enlargement was found.

\section{Pulmonary CT characteristics at reexamination}

At reexamination, the lung lesions of patients with moderate disease were significantly improved, especially lesions in the upper lobes of the lungs $(\mathrm{P}<0.05)$ (Table 2). Five patients had newly developed patchy GGOs with blurred borders in the lungs, and GGOs in 21 patients with moderate disease $(17.5 \%)$ were markedly improved $\left(\chi^{2}=6.495, \mathrm{P}<0.05\right)$. The absorption of consolidation shadows and parenchymal bands was slow, and some GGOs turned into consolidation shadows (Figure 1). The consolidation shadows in 56 patients with moderate disease $(46.7 \%)$ had substantially improved $\left(\chi^{2}=11.526, \mathrm{P}<0.05\right)$, and parenchymal bands in 85 patients with moderate disease $(70.8 \%)$ were notably reduced $\left(\chi^{2}=18.813, \mathrm{P}<0.05\right)$ (Figure 2$)$. The absorption of IST and subpleural lines was fast; IST in 49 patients with moderate disease $(40.8 \%)$ had significantly improved $\left(\chi^{2}=31.790, \mathrm{P}<0.05\right)$ (Figure 3$)$, and subpleural lines in 41 patients with moderate disease $(34.2 \%)$ were markedly reduced $\left(\chi^{2}=33.865, \mathrm{P}<0.05\right)$. Vascular enlargement in lesions in 12 patients with moderate disease $(10.0 \%)$ was considerably reduced $\left(\chi^{2}=6.128, \mathrm{P}<0.05\right)$, and air bronchograms in six patients with moderate disease $(5.0 \%)$ did not improve significantly $\left(\chi^{2}=1.583, \mathrm{P}>0.05\right)$ (Table 3).

At reexamination, the lung lesions in patients with severe disease had partially improved, especially in patients with multi-lobe involvement $\left(\chi^{2}=3.956, \mathrm{P}<0.05\right)$ (Table 2). 

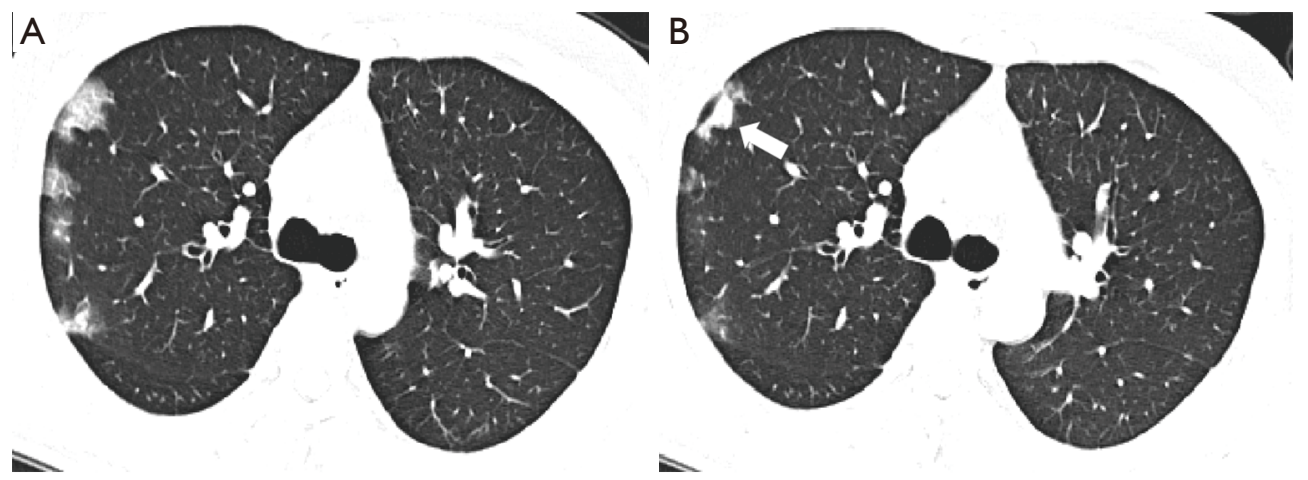

Figure 1 Coronavirus disease 2019 (COVID-19) with moderate disease. (A). At discharge, patchy ground-glass opacity (GGO) was scattered in the subpleural part of the right lung with a well-defined border. (B) At reexamination, these GGOs were smaller and denser than before, showing a consolidation shadow (arrow).

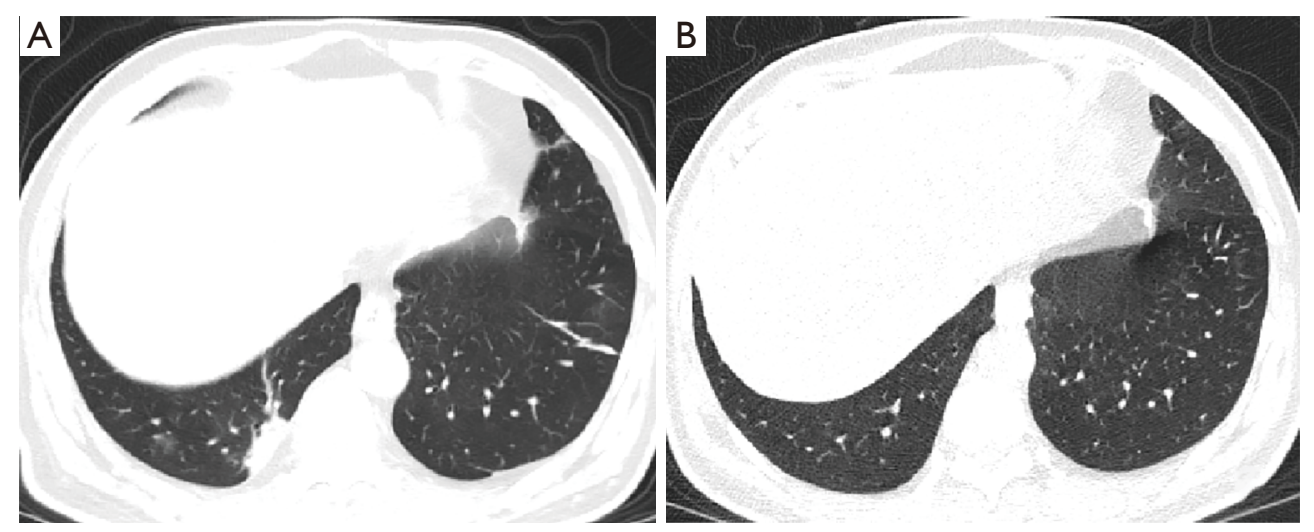

Figure 2 Coronavirus disease 2019 (COVID-19) with moderate disease. (A) At discharge, in the lower lobe of double lung, there were striplike consolidation shadow and fiber cord shadow with clear boundaries. (B) At reexamination, the lesions in the lower lobe of both lungs were completely absorbed.

However, the GGOs, consolidation shadows, IST, parenchymal bands, subpleural lines, vascular enlargement in lesions, and air bronchograms in patients with severe disease did not show significant changes at reexamination $(\mathrm{P}>0.05)$ (Table 4, Figure 4).

\section{Discussion}

Most COVID-19 patients have mild or moderate disease, however a small number of patients progress rapidly and develop severe and critical disease, making treatment difficult. The condition of COVID-19 patients is associated with many factors, including physique, underlying diseases, and treatment regimens. The condition of some patients might fluctuate, and they can even develop acute respiratory distress syndrome (ARDS) or multiple organ failure (MOF), which may be related to a cytokine storm $(9,10)$. The participants enrolled in this study were cured patients, including 120 patients with moderate disease and 20 patients with severe disease, and some had underlying comorbidities. Chen et al. (11) retrospectively analyzed 99 hospitalized patients and found that patients mainly presented with fever, cough, and related respiratory symptoms, accompanied by concomitant symptoms in other parts of the body. These findings are consistent with the results of the present study. In addition, some patients in this study had other symptoms, such as nausea, vomiting, and diarrhea, indicating that SARS-CoV-2 may also cause gastrointestinal wall damage and dysfunction, similar to the symptoms associated with SARS and Middle East 

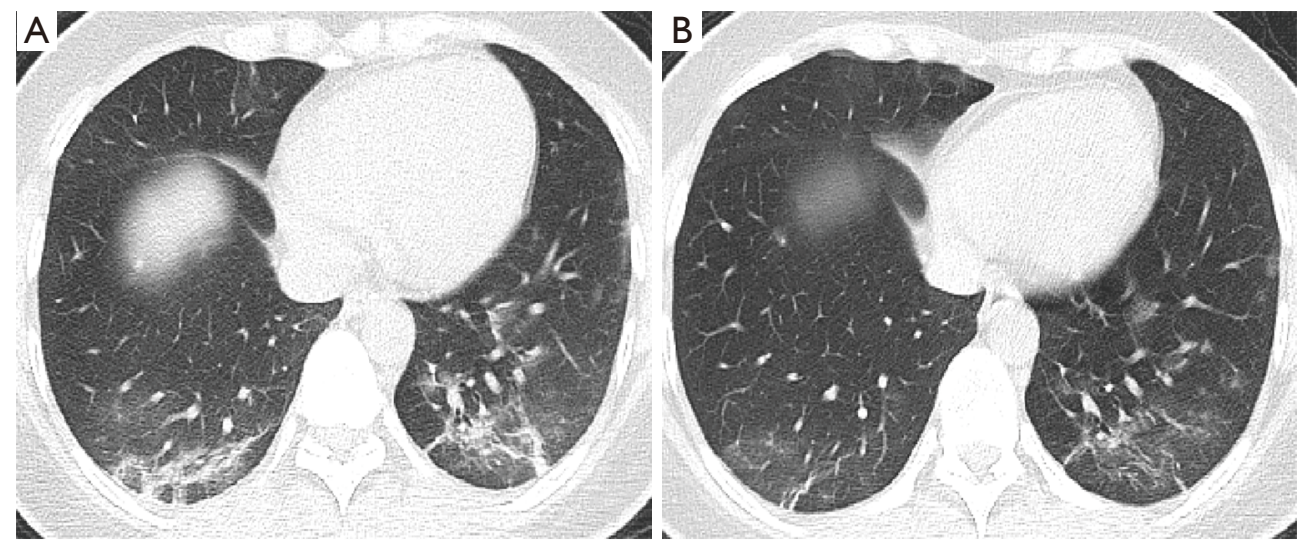

Figure 3 Coronavirus disease 2019 (COVID-19) with moderate disease. (A) At discharge, in the lower lobe of double lung, there were ground-glass opacity (GGO), interlobular septal thickening (IST), fiber cord shadow and vascular enlargement in lesions. (B) At reexamination, the lesions in the lower lobe of both lungs were more absorbed than before.

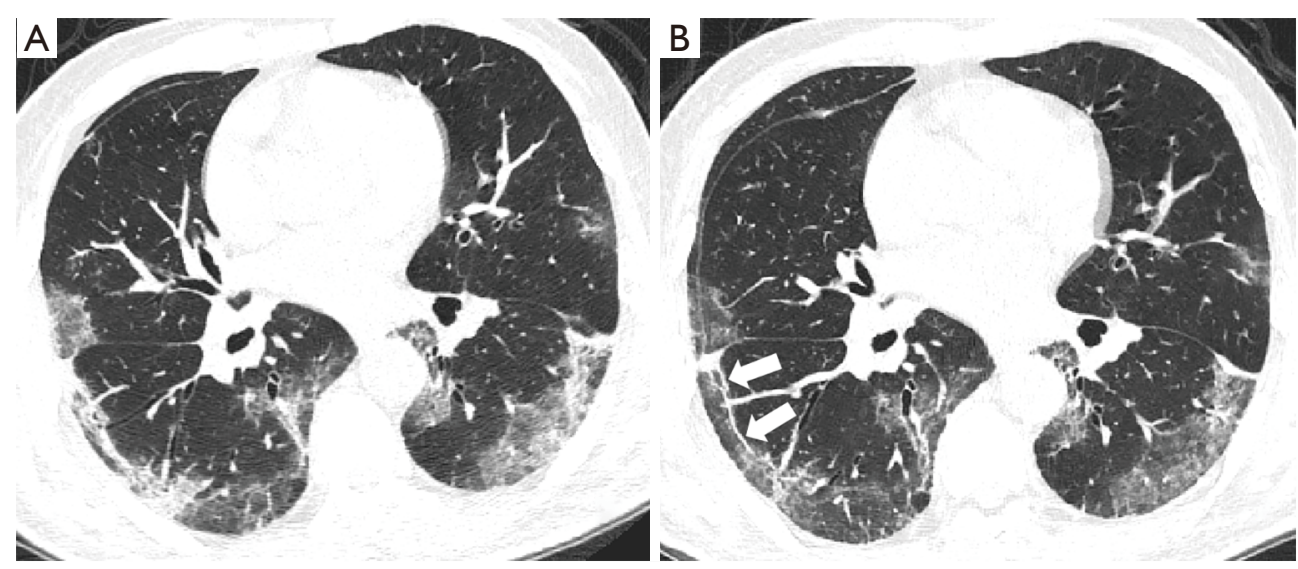

Figure 4 Coronavirus disease 2019 (COVID-19) with severe disease. (A) At discharge, multiple ground-glass opacities (GGOs) and partial consolidation were seen in the peripheral bands of both lungs. (B) At reexamination, the double lung lesions were more absorbed, and subpleural lines was seen (arrow).

respiratory syndrome (MERS) (12).

At the early stage of treatment, patients in this study had varying lung lesions with different ranges and degrees that were effectively treated. At discharge, 15 patients had no lung lesions. Studies have shown that the pathological characteristics of COVID-19 are very similar to those of SARS and MERS, and manifest as alveolar damage with cellular fibromyxoid exudates (13). Therefore, the absorption of lung lesions in COVID-19 patients is slow, especially in patients with severe disease and severe lung injury. In this study, 140 patients still had different degrees of lesions on lung CT images at discharge. Due to the small size of SARS-CoV-2, after inhalation through the airway, it mainly invades the bronchioles, causing bronchiolitis and peripheral inflammation, and spreads distally, invading lung tissues. SARS-CoV-2 tends to invade the periphery of both lungs with abundant capillaries, especially the lower lobes (14). Therefore, most lung lesions in patients in this study were located in the periphery and subpleural area of both lungs, which is consistent with the distribution characteristics of the initial lesions, and may also be related to the fact that SARS-CoV-2 mainly colonizes lower respiratory bronchioles and alveolar epithelial cells (15). In addition, this study found that the lesions mostly involved the lower lobes of the right lung, which is consistent with the characteristics of aspiration pneumonia. At 
reexamination, CT images showed that the absorption of lesions in the upper lobes of the lungs was relatively fast and that the absorption of the lesions in the lower lobes of the lungs was slow, which was due to the relatively severe damage in the lower lobes of the lungs.

In COVID-19 patients, pulmonary CT mainly shows multiple GGOs and consolidation shadows distributed along the bronchovascular bundle in both lungs, and IST is also observed in some cases (16). This is primarily caused by thickening of the alveolar wall, collapsed alveoli, and increased local blood flow. SARS-CoV-2 first invades the distal peribronchial interstitium below the lobular bronchioles. Lymphatic reflux in the central area of the lobules is centripetal and thus conducive to the formation of circular GGOs. Subsequently, SARS-CoV-2 gradually spreads to the entire secondary pulmonary lobules and surrounding lung tissues, forming patchy GGOs, whose size is consistent with that of the secondary pulmonary lobules. With the enlargement and fusion of the lesions, large patchy GGOs form. When the virus affects the interstitial substance around the lobules, GGOs often appear as stripe-like, which is due to the fact that the lymphatic circumfluence in this region involves peripheral drainage to the subpleural and interlobular septa. The lesions are close to the pleura and spread along the bilateral interlobular septa, causing the long axis of the lesions to be parallel to the pleura. Therefore, GGOs mostly manifest with round, patchy, large patchy, and striped morphologies. In this study, the GGOs in the patients were mainly caused by the gradual absorption and reduction in the original patchy and striped lesions. According to previous studies, the presence of GGOs suggests the existence of exudative lesions in the lungs, indicating that there might be a small amount of virus remaining in the patient. Therefore, monitoring the health of discharged patients is necessary (17). Studies have shown that it is still possible for convalescent patients to show positive for SARS-CoV-2 nucleic acid detection, but most of the repositive patients showed no deterioration in pulmonary CT findings. Continuous quarantine and close follow-up for convalescent patients are necessary to prevent possible relapse and spread of the disease to some extent (18).

In this study, five patients had newly developed GGOs in the lungs at reexamination, and the borders were ambiguous. Since the GGOs in the lungs of COVID-19 patients are mostly clear and straight, we believed that the newly developed GGOs were caused by other bacterial infections, and the newly developed GGOs improved after antibiotic treatment. In the remaining patients, GGOs decreased significantly, and some of them changed to consolidation shadows. Song et al. (19) considered pulmonary consolidation to be one of the indicators for the progression or exacerbation of COVID-19. In the absorption phase, consolidation shadows appear as a result of pulmonary interstitial fibrosis and alveolar collapse. However, the lesion range is smaller than in previous stages, the density is higher compared to the early and progressive stages, and air bronchospasms are rare. Therefore, reduced GGO range and increased density may indicate that a patient's condition has improved. SARS-CoV-2 enters the alveoli and easily binds to type II alveolar epithelial cells, causing reduced secretion of alveolar surfactants, which leads to adhesive atelectasis. These lesions are also classified as consolidation shadows and are consistent with the clinical symptoms of hypoxemia and dyspnea. This may also be one of the reasons for the slow absorption of lung consolidation shadows in this study, and nearly half of the patients still had consolidation shadows at reexamination. COVID-19 pulmonary lesions are often associated with enlarged vascular bundles (20), which are manifestations of vasculitis, perivasculitis, and hyperemia resulting from lesions involving the pulmonary arteries, which is mainly due to the acute inflammatory response. A small amount of vascular enlargement in lesions was observed in the cured COVID-19 patients in this study, which may be attributable to perivascular interstitial thickening, although this gradually reduced with improvement of the disease.

IST on CT images often manifests as short-line highdensity shadows extending to the pleura, also known as "septal shadows". It has been reported that IST can occur in the early stage of COVID-19 and throughout the entire disease course $(21,22)$, suggesting that COVID-19 can involve the interstitial substance, making it fibrous. Previous studies have shown that pulmonary interstitial fibrosis is severe in SARS patients and that intralobular interstitium, IST, and subpleural lines remain in the lobules after discharge $(23,24)$. However, recent autopsy results showed that the pulmonary fibrosis in a COVID-19 patient was not as severe as that in SARS patients and that the exudative response was more obvious compared to SARS patients (25). This study found that in COVID-19 patients, there were varying degrees of absorption of and reduction in IST and parenchymal bands, suggesting that the degree of interstitial lung fibrosis in COVID-19 did not reach the level of complete fibrosis and that there was still a recovery potential. Whether parenchymal bands indicate irreversible 
fibrosis and affect the recovery of lung function and the clinical prognosis of patients remains to be investigated in studies with large sample sizes and long-term follow-up. To further explore CT features of pulmonary fibrosis recovery potential. Subpleural lines appear as a linear shadow parallel to the pleura and are commonly seen as striped lesions in the subpleural area of the lung. The formation mechanism may involve the following: the interlobular septum at the inner and outer ends of the lesion recovers relatively quickly, and the striped septal shadow remains in the middle of the lesion. In this study, at reexamination, subpleural lines existed in only $34.2 \%$ of the patients with moderate disease, while the number of patients with severe disease with subpleural lines was higher. Therefore, subpleural lines in the periphery of the lungs suggest that the lung lesions in COVID-19 patients might be in the absorption phase, and the patient has a good prognosis. According to the guidelines, the CT features of the lung that meet the discharge criteria is the acute exudative lesions were significantly improved. In this study, it was found that the changes of lung lesions were not obvious in the patients with severe disease, indicating that severe disease caused severe lung damage and slow recovery. Therefore, the frequency of return visit should be increased, and the changes of lungs should be observed with emphasis.

In summary, pulmonary CT can directly reflect the post-treatment trends of pulmonary lesions in COVID-19 patients, providing a reference for the clinical evaluation of treatment efficacy and the prognosis of patients. However, this study also had limitations that should be noted. Firstly, the sample size was relatively small; large-sample and multicenter studies are required to validate the results. Secondly, only the results of one CT reexamination were analyzed; multiple follow-up visits are needed to acquire more data.

\section{Acknowledgments}

We thank all participants in this study.

Funding: None.

\section{Footnote}

Reporting Checklist: The authors have completed the MDAR checklist. Available at http://dx.doi.org/10.21037/apm-202532

Data Sharing Statement: Available at http://dx.doi. org/10.21037/apm-20-2532

Conflicts of Interest: All authors have completed the ICMJE uniform disclosure form (available at http://dx.doi. org/10.21037/apm-20-2532). The authors have no conflicts of interest to declare.

Ethical Statement: The authors are accountable for all aspects of the work in ensuring that questions related to the accuracy or integrity of any part of the work are appropriately investigated and resolved. All procedures performed in this study involving human participants were in accordance with the Declaration of Helsinki (as revised in 2013). This study was approved by the Ethics Committee of First Affiliated Hospital of Kunming Medical University, and confirm that informed consent was obtained from all patients.

Open Access Statement: This is an Open Access article distributed in accordance with the Creative Commons Attribution-NonCommercial-NoDerivs 4.0 International License (CC BY-NC-ND 4.0), which permits the noncommercial replication and distribution of the article with the strict proviso that no changes or edits are made and the original work is properly cited (including links to both the formal publication through the relevant DOI and the license). See: https://creativecommons.org/licenses/by-nc-nd/4.0/.

\section{References}

1. Li Q, Guan X, Wu P, et al. Early transmission dynamics in Wuhan, China, of novel coronavir-infected pneumonia. N Engl J Med 2020;382:1199-207.

2. Rothe C, Schunk M, Sothmann P, et al. Transmission of 2019-nCoV infection from an asymptomatic contact in Germany. N Engl J Med 2020;382:970-1.

3. National Health Commission of the People's Republic of China. Diagnosis and treatment of COVID-19 (7th edition): Letter from General Office of the National Health Commission of China (2020) No. 184 (S). Available online: http://www.nhc.gov.cn/yzygj/s7653p/202003/46c9 294a7dfe4cef80dc7f5912eb1989.shtml

4. Fang $\mathrm{Y}$, Zhang $\mathrm{H}, \mathrm{Xu} \mathrm{Y}$, et al. CT manifestations of two cases of 2019 novel coronavirus (2019-nCoV) pneumonia. Radiology 2020;295:208-9.

5. $\mathrm{Li} \mathrm{K}, \mathrm{Wu} \mathrm{J}, \mathrm{Wu} \mathrm{F}$, et al. The clinical and chest CT features associated with severe and critical COVID-19 pneumonia. Invest Radiol 2020;55:327-31. 
6. $\mathrm{Xu} \mathrm{X,} \mathrm{Yu} \mathrm{C,} \mathrm{Qu} \mathrm{J,} \mathrm{et} \mathrm{al.} \mathrm{Imaging} \mathrm{and} \mathrm{clinical} \mathrm{features} \mathrm{of}$ patients with 2019 novel coronavirus SARS-CoV-2. Eur J Nucl Med Mol Imaging 2020;47:1275-80.

7. Ji G, Huang M, Zhang Q, et al. The findings and dynamic changes in CT images of patients with COVID-19. Chinese Journal of Medical Imaging Technology 2020;36:242-7.

8. Wang J, Liu J, Wang Y, et al. Dynamic changes of chest CT imaging in patients with corona virus disease-19. Zhejiang Da Xue Xue Bao Yi Xue Ban 2020;49:191-7.

9. Gupta KK, Khan MA, Singh SK. Constitutive Inflammatory Cytokine Storm: A Major Threat to Human Health. J Interferon Cytokine Res 2020;40:19-23.

10. Wang D, Hu B, Hu C, et al. Clinical Characteristics of 138 Hospitalized Patients With 2019 Novel CoronavirusInfected Pneumonia in Wuhan, China. JAMA 2020;323:1061-9.

11. Chen N, Zhou M, Dong X, et al. Epidemiological and clinical characteristics of 99 cases of 2019 novel coronavirus pneumonia in Wuhan, China: a descriptive study. Lancet 2020;395:507-13.

12. Wong G, Liu W, Liu Y, et al. MERS, SARS, and Ebola: the role of super-spreaders in infectious disease. Cell Host Microbe 2015;18:398-401.

13. Xu Z, Shi L, Wang Y, et al. Pathological findings of COVID-19 associated with acute respiratory distress syndrome. Lancet Respir Med 2020;8:420-2.

14. Zhu N, Zhang D, Wang W, et al. A Novel Coronavirus from Patients with Pneumonia in China, 2019. N Engl J Med 2020;382:727-33.

15. Huang C, Wang Y, Li X, et al. Clinical features of patients infected with 2019 novel coronavirus in Wuhan, China. Lancet 2020;395:497-506.

16. Chinese Society of Radiology, Chinese Medical

Cite this article as: Yang Y, Zhang Z, Jiang Y, Li Z, Yang H, Li Z, Li X, Yang J, Zhang J, Peng Y, Luo L, Chang W, Zhao W, Wu Y, Ma C, Wang S, Wen Y, Pu F, Jiang X, Shen L, Zhan Z, Jin Y, Li Q, Yi W, Xie Y, Tang Y, Duan H, Yang K, Su Y, Wu C, Luo Z, Wang P, Hong M, Yang S, Bai W, Yu G, Liu S, Nie Z, Song G, Guo D, He X, Chen Z, Xue K, Ma J, Wu Y, Yang X, He B, Wang K, Pi J. Dynamic analysis of pulmonary computed tomography (CT) characteristics in cured coronavirus disease 2019 (COVID-19) patients. Ann Palliat Med 2021;10(2):2062-2071. doi: 10.21037/apm-20-2532
Association. Radiologic diagnosis of COVID-19:

Recommendations from the Chinese Society of Radiology (1st edition). Chinese Journal of Radiology 2020;54:E001.

17. Li X, Wang H, Zhu J, et al. Dynamic imaging observation of intrapulmonary lesions in cured COVID-19 patients (imported). Chinese Journal of Radiology 2020;54:E012.

18. Zhu H, Fu L, Jin Y, et al. Clinical features of COVID-19 convalescent patients with re-positive nucleic acid detection. J Clin Lab Anal 2020;34:e23392.

19. Song F, Shi N, Shan F, et al. Emerging 2019 Novel Coronavirus (2019-nCoV) Pneumonia. Radiology 2020;295:210-17.

20. Yu C, Qu J, Zhang L, et al. High resolution CT findings and clinical characteristics of COVID-19 patients in Guangzhou area. Chinese Journal of Radiology 2020;54:E010.

21. Guan H, Xiong Y, Shen N, et al. Clinical and thin-section CT characteristics of patients with 2019-nCoV-pneumonia in Wuhan. Radiologic Practice 2020;35:125-30.

22. Li Y, Xia L. Coronavirus Disease 2019 (COVID-19): Role of Chest CT in Diagnosis and Management. AJR Am J Roentgenol 2020;214:1280-6.

23. Du X, Yu W, Wang S, et al. Clinical and imaging analyses of patients with SARS. Chinese Journal of Radiology 2003;37:780-3.

24. Brand JM, Haagmans BL, Riel D, et al. The pathology and pathogenesis of experimental severe acute respiratory syndrome and influenza in animal models. J Comp Path 2014;151:83-112.

25. Liu X, Wang R, Qu G, et al. Autopsy report for a deceased COVID-19 patient. Journal of Forensic Medicine 2020;36:1-3.

(English Language Editor: A. Kassem) 